\title{
Duplik zu Fracheboud et al.
}

\section{Rolf Ritschard}

Lic. phil., Soziologe

\footnotetext{
* Die Literatur findet sich unter www.saez.ch $\rightarrow$ Aktuelle Ausgabe oder $\rightarrow$ Archiv $\rightarrow 2016 \rightarrow 3$

Die Begriffe Prävalenz- und Inzidenzscreening kommen in der Literatur kaum vor, ich habe sie in den europäischen Leitlinien [4] einmal gefunden. Sie verwirren mehr, als dass sie der Transparenz zum Durchbruch verhelfen. Dass beim Screening auch gewisse technische und organisatorische Massnahmen Qualitätskriterien, die in den Leitlinien festgelegt sind, genügen müssen, scheint mir selbstverständlich zu sein Im Abstract werden sogar nur Intervallkarzinome zur Berechnung der Prävalenzrate erwähnt

${ }^{4}$ Die Schätzung des Bundesamtes für Statistik war mit 72000 etwas zu hoch
}

Um einer Sprachverwirrung zu entgehen, wird im Folgenden konsequent von Erstscreenings und Folgescreenings sowie von den in der medizinischen Literatur gebräuchlichen Begriffen Prävalenz und Inzidenz gesprochen ${ }^{1}$. Prävalenz wird folgendermassen definiert: «Die Prävalenz einer bestimmten Krebserkrankung bezeichnet die Anzahl der Personen innerhalb einer festgelegten Bevölkerungsgruppe, bei denen diese Krebsart diagnostiziert wurde und die zum Zeitpunkt der Untersuchung noch lebten" [1]*.

Und Inzidenz folgendermassen: «Inzidenz beschreibt die Anzahl der Neuerkrankungen, die innerhalb einer festgelegten Bevölkerungsgruppe in einem bestimmten Zeitraum auftreten. Sie wird als absolute Anzahl der Neuerkrankungen pro Jahr oder als Rate pro 100000 Einwohner pro Jahr angegeben» [2].

«Beobachtungsstudien mit Daten aus laufenden Programmen erlauben es, gewisse Performance-Indikatoren zu berechnen, sind aber äusserst heikel, wenn mit ihnen der reale Effekt einer Screening-Massnahme eruiert werden soll» [3]. Ich habe es trotzdem versucht, weil die Qualität einer Massnahme letztlich nur an der Wirkung bzw. am Nutzen und Schaden evaluiert werden kann. Die europäischen Leitlinien [4] sind für diese Art der Qualitätsevaluation methodisch fragwürdig. Es wird eine zu erwartende Inzidenz ohne Screening benützt, bei der unbekannt ist, wie sie eruiert wird. Wie auf dieser schwachen Grundlage Brustkrebsentdeckungsraten berechnet werden können, ist nicht nachvollziehbar². Obwohl die Leitlinien von Inzidenzraten ohne Screening ausgehen, berechnen die Autoren in ihrer Entgegnung die Prävalenzrate. Vielleicht möchten sie nachweisen, dass die Prävalenzrate tiefer ist als die von mir verwendete von $1 \%$. Wieso die Prävalenzrate allerdings mit der Addition von Brustkrebsentdeckungraten und der Häufigkeit von Intervallkarzinomen ${ }^{3}$ berechnet wird, ist nicht nachvollziehbar und widerspricht der üblichen Definition von Prävalenz. Dazu kommt, dass von sehr kleinen Fallzahlen ausgegangen wird, so dass die Berechnungen höchst fragwürdig sind. Die von mir verwendete Prävalenzrate von $1 \%$ beruht auf einer grossen Anzahl von Studien und von 650000 untersuchten Frauen [5]. In der Schweiz sieht die Prävalenz folgendermassen aus: Im Jahre 2000 waren etwa 45900 Frauen brustkrebskrank, im Jahre 2010 rund 65000 [6]. Dies ergibt, gemessen an der gesamten weiblichen Bevölkerung, eine Prävalenzrate von 1,2 bzw. 1,6\% ${ }^{4}$. Diese Prävalenz- raten sind eher zu tief, da in der weiblichen Bevölkerung auch Kinder und Jugendliche mitgezählt sind. Somit sollte meine Berechnung der Prävalenzrate nachvollziehbar sein, mit $1 \%$ vermutlich für die Schweiz eher etwas zu tief.

Die Autoren meinen, dass Frauen, bei denen früher eine Brustkrebsdiagnose gestellt worden war, aus offensichtlichen Gründen gar nicht mehr am Screening teilnehmen. Diese Hypothese wird nicht belegt. Dass die Hälfte der Brustkrebsüberlebenden über 70 Jahre alt sein dürfte, bezieht sich auf alle Krebsarten [7], stimmt aber für Brustkrebs nicht: Es gibt im Jahre 201026667 Frauen zwischen 0 und 69 Jahren mit einer Brustkrebsdiagnose seit 0-10 Jahren. Nur 15137 Frauen mit dieser Diagnose seit 0-10 Jahren sind über 70 Jahre alt [6]. Die etwas verwirrende Argumentation der Autoren über den Anteil invasiver Karzinome mit einem Durchmesser von $15 \mathrm{~mm}$ oder weniger kann am Beispiel des deutschen Jahresberichts Evaluation 2012 [8] näherungsweise geklärt werden (S. 20): Mit dem Screening (im Vergleich zu ohne Screening) erhöhen sich dort die Anteile der entdeckten invasiven Karzinome bis $20 \mathrm{~mm}$ von $49 \%$ auf $82 \%$, die Karzinome über $20 \mathrm{~mm}$ verringern sich von $44 \%$ auf $18 \%$, was im Rahmen der europäischen Leitlinien liegt. Dies mag auch für den Evaluationsbericht in der Schweiz gelten.

Dass es sich bei den kleineren invasiven Karzinomen dennoch in den meisten Fällen um verpasste Diagnosen handeln könnte, liegt in folgender biologischer Regel begründet: Bis ein Karzinom entdeckt werden kann, müssen mindestens 30 Mitosen stattgefunden haben, das ergibt $2^{30}$ oder rund 1 Milliarde Zellen, entsprechend einem Volumen von rund $1 \mathrm{~cm}^{3} \mathrm{Krebs}$ [9]. Es gibt wohl kaum Karzinome, die innerhalb von 2 Jahren dermassen explosiv wachsen.

Die von den Autoren der Replik in Frage gestellten Zahlen sind meiner Meinung nach leicht belegbar. Ein Teil des Problems dürfte wohl in der unscharfen Begriffsdefinition liegen. Zusammenfassend kann festgehalten werden, dass die Qualität der Screeningprogramme im Hinblick auf deren Wirkung sowie bezüglich Nutzen und Schaden des Screenings sich mit den Indikatoren der Leitlinien nicht nachweisen lässt. Für eine informierte Entscheidung der Frauen ist eine so evaluierte Qualität nutzlos. Sie dient eher der Werbung.

Danksagung

Ich danke Dr. med. Jürg Kuoni für die kritische Durchsicht der Duplik. 


\section{Literatur}

1 www.nicer.org/de/statistiken-atlas/krebspraevalenz/

2 www.nicer.org/de/statistiken-atlas/krebsinzidenz

3 Jüni P, Erlanger T, Zwahlen M. Replik zum Beitrag «Swiss Medical Board - cui bono?». Schweiz Ärztezeitung. 2014;95(46):1726-8.

4 European guidelines for quality assurance in breast cancer screening and diagnosis (Fourth Edition); 2006.

5 Cochrane 4. Juni 2013: http://onlinelibrary.wiley.com/ doi/10.1002/14651858.CD001877.pub5/full
6 Nicer. Prevalence of breast cancer in Switzerland. www.23nicer. org/de/statistiken-atlas/krebspraevalenz/

7 Lorez M, Heusser R, Arndt V. Prevalence of Cancer Survivors in Switzerland. Schweizer Krebsbulletin. 2014;287.

8 Jahresbericht Evaluation 2012. Deutsches Mammographie-Scree ning-Programm. Kooperationsgemeinschaft. Mammographie, Berlin; August 2015.

9 Weinberg R. One Renegade Cell: How Cancer Begins. Basic Books; 1999. 